\author{
Y. STOYAN (Kharkov) \\ J. TERno ${ }^{\dagger}$ (Dresden) \\ M. GIL (Kharkov) \\ T. ROMANOva (Kharkov) \\ G. Scheithauer (Dresden)
}

\title{
CONSTRUCTION OF A $\Phi$-FUNCTION FOR TWO CONVEX POLYTOPES
}

Abstract. The analytical description of $\Phi$-functions for two convex polytopes is investigated. These $\Phi$-functions can be used for mathematical modelling of packing problems in the three-dimensional space. Only translations of the polytopes are considered. The approach consists of two stages. First the 0-level surface of a $\Phi$-function is constructed, and secondly, the surface is extended to get the $\Phi$-function. The method for constructing the 0-level surface is described in detail.

1. Introduction. Cutting and packing problems are of wide interest in practical applications as well as in scientific investigations. There is a long stream of publications concerning such problems. But only a small part of them is devoted to three-dimensional (3D) placement problems for irregular (but convex) polytopes. For an annotated review on cutting and packing problems we refer the reader to [2]. A lot of published work is also classified in $[1]$.

In this paper we address in particular cutting and packing problems where real, arbitrarily shaped objects are to be placed in a larger region, or cut out from it.

2000 Mathematics Subject Classification: 90B06, 90C11, 90C26.

Key words and phrases: $\Phi$-function, cutting and packing, convex polytope.

Research of Y. Stoyan was partially supported by the German Research Society (DFG, grant TE 207/7, 436 UKR 113/42/0).

$\dagger$ In the final stage of this work, Prof. Dr. Johannes Terno passed away after a long serious illness. He will stay in our minds for ever. 
One of the most important topics when (3D) placement problems have to be modelled (cf. [2], [11], [12]) is the description and handling of the interaction between pairs of objects which have to be placed within a larger region. As is known, one of the possibilities for such a modelling is to construct a function which characterizes these interactions in the following sense. The values of the function should distinguish between the following three situations: the two objects intersect each other, they do not have common points, or they are tangent. A function with those properties will be called a $\Phi$-function. Moreover, a $\Phi$-function should give some information on the distance between the two objects being placed.

$\Phi$-functions are very helpful in algorithms for solving packing and cutting problems with arbitrarily shaped objects. Especially in algorithms where one tries to improve a given solution (packing pattern) by local optimization (small movements of the objects), the interaction of all objects has to be controlled.

Based on work for general placement problems ([9], [10]) we present a construction of a $\Phi$-function for a pair of convex $3 \mathrm{D}$ polytopes. After describing the concept of $\Phi$-function and investigating their properties (Section 2), we consider some two-dimensional cases in Section 3. Then, in Section 4, we construct the 0-level surface of a $\Phi$-function for a pair of convex $3 \mathrm{D}$ polytopes. In Section 5, the computation of the corresponding $\Phi$-function is discussed. An algorithmic description of the approach is presented in Section 6 , followed by a detailed algorithm for a special subcase (Section 7). Some final remarks are also given.

2. The concept of $\Phi$-functions. In a packing and cutting formulation, the aim is to construct a function which depends on the mutual location of two polytopes $T_{1}$ and $T_{2}$ and whose values give a numerical evaluation for the following three cases:

1. $T_{1}$ and $T_{2}$ intersect, i.e. they have common interior points;

2. $T_{1}$ and $T_{2}$ are disjoint, i.e. they do not have common points;

3. $T_{1}$ and $T_{2}$ are in contact, i.e. they have only common frontier points.

Let int $T$ denote the interior of a set $T$, and fr $T$ the frontier of $T$. Then the three cases are:

1. $\operatorname{int} T_{1} \cap \operatorname{int} T_{2} \neq \emptyset$,

2. $T_{1} \cap T_{2}=\emptyset$,

3. int $T_{1} \cap \operatorname{int} T_{2}=\emptyset$ and $\operatorname{fr} T_{1} \cap$ fr $T_{2} \neq \emptyset$.

Moreover, it is desirable that the required function values be a measure of intersection of $T_{1}$ and $T_{2}$ in the first case, and define or at least estimate the distance between the polytopes in the second case. 
In this paper, "placement" means "translating by some vector". Each object $T_{i}(i=1,2)$ is given with respect to a fixed coordinate system, called its eigen coordinate system. The origin of the system is also called the pole of $T_{i}$. The object $T_{i}$ translated by a vector $u_{i}=\left(x_{i}, y_{i}, z_{i}\right)$ can be described by

$$
T_{i}\left(u_{i}\right):=\left\{X \in \mathbb{R}^{3}: X=u_{i}+Y, Y \in T_{i}\right\} .
$$

$u_{i}=\left(x_{i}, y_{i}, z_{i}\right)$ is also called the vector of placement parameters $x_{i}, y_{i}, z_{i}$ of $T_{i}(i=1,2)$. As usual, $u_{i}$ specifies the location of the origin of the eigen coordinate system of $T_{i}$ with respect to the coordinate system of the region where the objects have to be placed. In [9] $\Phi$-functions are introduced in order to formalize the description of the interaction of a pair of polytopes $T_{1}$ and $T_{2}$.

Definition 1. A continuous function $\Phi: \mathbb{R}^{6} \rightarrow \mathbb{R}$ is called a $\Phi$-function of $T_{1}$ and $T_{2}$ if it has the following properties:

(1) $\Phi\left(u_{1}, u_{2}\right) \begin{cases}>0 & \text { if } T_{1}\left(u_{1}\right) \cap T_{2}\left(u_{2}\right)=\emptyset, \\ =0 & \text { if } \operatorname{int} T_{1}\left(u_{1}\right) \cap \operatorname{int} T_{2}\left(u_{2}\right)=\emptyset, \operatorname{fr} T_{1}\left(u_{1}\right) \cap \operatorname{fr} T_{2}\left(u_{2}\right) \neq \emptyset, \\ <0 & \text { if } \operatorname{int} T_{1}\left(u_{1}\right) \cap \operatorname{int} T_{2}\left(u_{2}\right) \neq \emptyset .\end{cases}$

Let $\gamma:=\left\{\left(u_{1}, u_{2}\right) \in \mathbb{R}^{6}: \Phi\left(u_{1}, u_{2}\right)=0\right\}$ denote the 0-level surface of the $\Phi$-function. Furthermore, let

$$
G=\left\{\left(u_{1}, u_{2}\right): \operatorname{int} T_{1}\left(u_{1}\right) \cap \operatorname{int} T_{2}\left(u_{2}\right)=\emptyset\right\}
$$

be the set of feasible placement points (or translation vectors) $\left(u_{1}, u_{2}\right)$, i.e. the set of all points such that the two translated polytopes do not intersect.

Definition 2. A $\Phi$-function $\Phi$ is said to be normalized if $\Phi\left(u_{1}, u_{2}\right)$ equals the Euclidean distance between $T_{1}\left(u_{1}\right)$ and $T_{2}\left(u_{2}\right)$ for all $\left(u_{1}, u_{2}\right) \in G$.

A $\Phi$-function of $T_{1}$ and $T_{2}$ can be obtained by a natural approach, namely the following optimization problem has to be solved for any $\left(u_{1}, u_{2}\right) \in G$ :

$$
\Psi\left(u_{1}, u_{2}\right)=\min _{X_{1}, X_{2} \in \mathbb{R}^{3}}\left\{\left\|X_{1}-X_{2}\right\|: f_{1}\left(X_{1}-u_{1}\right) \leq 0, f_{2}\left(X_{2}-u_{2}\right) \leq 0\right\},
$$

where $f_{i}(X)=0$ is the equation of fr $T_{i}$ :

$$
f_{i}(X)=\left\{\begin{array}{ll}
>0 & \text { if } X \notin T_{i}, \\
\leq 0 & \text { if } X \in T_{i},
\end{array} \quad i=1,2 .\right.
$$

$\|\cdot\|$ denotes the Euclidean norm.

Solving problem (2) can be significantly simplified if either $u_{1}=0$ or $u_{2}=0$, i.e. either $T_{1}$ or $T_{2}$ is considered to be fixed. Then one of the two problems has to be solved:

$$
\begin{array}{r}
\Psi_{1}\left(u_{1}\right)=\min _{X_{1}, X_{2} \in \mathbb{R}^{3}}\left\{\left\|X_{1}-X_{2}\right\|: f_{1}\left(X_{1}-u_{1}\right)=0, f_{2}\left(X_{2}\right)=0\right\} \\
\forall\left(u_{1}, 0\right) \in G,
\end{array}
$$


or

$$
\begin{array}{r}
\Psi_{2}\left(u_{2}\right)=\min _{X_{1}, X_{2} \in \mathbb{R}^{3}}\left\{\left\|X_{1}-X_{2}\right\|: f_{1}\left(X_{1}\right)=0, f_{2}\left(X_{2}-u_{2}\right)=0\right\} \\
\forall\left(0, u_{2}\right) \in G .
\end{array}
$$

Then the function $\Psi\left(u_{1}, u_{2}\right),\left(u_{1}, u_{2}\right) \in G$, is either $\Psi\left(u_{1}, u_{2}\right):=\Psi_{1}\left(u_{1}-u_{2}\right)$ or $\Psi\left(u_{1}, u_{2}\right):=\Psi_{2}\left(u_{2}-u_{1}\right)$.

The function $\Psi\left(u_{1}, u_{2}\right)$ specifies the distance between $T_{1}\left(u_{1}\right)$ and $T_{2}\left(u_{2}\right)$. Hence, $\Psi\left(u_{1}, u_{2}\right) \geq 0$ for all $\left(u_{1}, u_{2}\right) \in G$. Thus a $\Phi$-function can be constructed as a continuous extension of $\Psi\left(u_{1}, u_{2}\right)$ onto the entire space $\mathbb{R}^{6}$ so that $\left.\Phi\left(u_{1}, u_{2}\right)\right|_{G}=\Psi\left(u_{1}, u_{2}\right)$ and $\Phi\left(u_{1}, u_{2}\right)<0$ for all $\left(u_{1}, u_{2}\right) \in \mathbb{R}^{6} \backslash G$.

It is evident that the extension of $\Psi\left(u_{1}, u_{2}\right)$ can be realized in an infinite number of ways. However the extension should be natural, simple and as a rule, it should satisfy some additional requirements. For example, a $\Phi$-function should be such that $\left|\nabla \Phi\left(u_{1}, 0\right)\right|$ is constant for (almost) all $\left(u_{1}, 0\right) \in \mathbb{R}^{6} \backslash G$, or smooth (almost) everywhere on $\mathbb{R}^{6} \backslash G$. In addition, the construction of a $\Phi$-function should not be more complex than the construction of a $\Psi$-function. In this paper, the function $\Phi\left(u_{1}, u_{2}\right)$ is constructed by solving one the problems (2), (4) or (5), and by extending $\Psi\left(u_{1}, u_{2}\right)$ so that $\left|\nabla \Phi\left(0, u_{2}\right)\right|_{\mathbb{R}^{6} \backslash G} \mid\left(\right.$ or $\left.\left|\nabla \Phi\left(u_{1}, 0\right)\right|_{\mathbb{R}^{6} \backslash G} \mid\right)$ is constant almost everywhere.

A $\Phi$-function $\Phi\left(u_{1}, u_{2}\right)$ constructed in this manner has certain properties which will be required later.

1. By definition,

$$
\Phi\left(u_{1}, u_{2}\right)=\Phi\left(u_{1}-u_{2}, 0\right)=\Phi\left(0, u_{2}-u_{1}\right) \quad \forall\left(u_{1}, u_{2}\right) \in G .
$$

2. The surface $\gamma_{12}=\left\{u_{2} \in \mathbb{R}^{3}: \Phi\left(0, u_{2}\right)=0\right\}$ is congruent to the frontier of the so-called Minkowski sum ([4])

$$
T_{12}\left(u_{2}\right)=T_{1}(0)+(-1) T_{2}\left(u_{2}\right)
$$

provided that $T_{1}$ and $T_{2}$ do not have precise entrances (i.e. $T_{i}=\operatorname{cl}\left(\operatorname{int} T_{i}\right)$, $i=1,2$, where $\operatorname{cl} T$ denotes the closure of $T$; see [10]). Hence,

$$
\gamma_{12} \cong \operatorname{fr}\left(T_{12}\left(u_{2}\right)\right)=\operatorname{fr}\left(\left\{X_{1}-X_{2}: X_{1} \in T_{1}(0), X_{2} \in T_{2}\left(u_{2}\right)\right\}\right) .
$$

Analogously, defining $T_{21}\left(u_{1}\right)=T_{2}(0)+(-1) T_{1}\left(u_{1}\right)$ and $\gamma_{21}:=\left\{u_{1} \in \mathbb{R}^{3}\right.$ : $\left.\Phi\left(u_{1}, 0\right)=0\right\}$, we have

$$
\gamma_{21} \cong \operatorname{fr}\left(\left(T_{21}\left(u_{1}\right)\right)=\operatorname{fr}\left(\left\{X_{2}-X_{1}: X_{1} \in T_{1}\left(u_{1}\right), X_{2} \in T_{2}(0)\right\}\right) .\right.
$$

It should be noted that the sets $\gamma_{12}$ and $\gamma_{21}$ are known to be hodographs of functions for dense placement of objects ([13], [14]).

3. The surfaces

$$
\left\{u \in \mathbb{R}^{3}: \Phi(u, 0)=\varrho\right\} \quad \text { and } \quad\left\{u \in \mathbb{R}^{3}: \Phi(0, u)=\varrho\right\}
$$


are centrally symmetric to each other for any $\varrho \geq 0$, i.e.

$$
v \in\left\{u \in \mathbb{R}^{3}: \Phi(u, 0)=\varrho\right\} \Leftrightarrow-v \in\left\{u \in \mathbb{R}^{3}: \Phi(0, u)=\varrho\right\} .
$$

4. In particular, for $\varrho=0$ we have

$$
\gamma_{12}=\left\{u \in \mathbb{R}^{3}: \Phi(0, u)=0\right\}=-\left\{u \in \mathbb{R}^{3}: \Phi(u, 0)=0\right\}=-\gamma_{21},
$$

i.e. the surfaces $\gamma_{12}$ and $\gamma_{21}$ are mutually centrally symmetric.

5 . If $T_{1}$ and $T_{2}$ mutually are centrally symmetric sets then there exists a $\Phi$-function $\Phi$ such that $\Phi(u, 0)=\Phi(0, u)$ for all $(u, 0) \in G$.

6. If $T_{1}$ and $T_{2}$ are polytopes then one can always find a piecewise linear $\Phi$-function for them.

7. If $T_{1}$ and $T_{2}$ are nonempty convex polytopes then $\gamma_{12}$ and $\gamma_{21}$ are the frontiers of nonempty convex polytopes $T_{12}(0)$ and $T_{21}(0)$, respectively.

In order to compute $\Psi$ by solving (2), (4) or (5), in general a Lagrangian approach, the complete differential method or other methods for nonlinear constraint optimization problems can be used. But in some special cases these problems can be solved in explicit form. For example,

$$
\begin{aligned}
\Phi\left(u_{1}, u_{2}\right) & =\left\|u_{2}-u_{1}\right\|-\left(R_{1}+R_{2}\right) \\
& =\sqrt{\left(x_{2}-x_{1}\right)^{2}+\left(y_{2}-y_{1}\right)^{2}+\left(z_{2}-z_{1}\right)^{2}}-\left(R_{1}+R_{2}\right)
\end{aligned}
$$

is a normalized $\Phi$-function of two solid spheres with radii $R_{1}$ and $R_{2}$ and centers $u_{i}=\left(x_{i}, y_{i}, z_{i}\right), i=1,2$, respectively.

However, it should be noted that if the functions $f_{1}, f_{2}$ in (3) are not smooth, or are curves of order greater than two, then any of the problems (2), (4) or (5) has to be solved by iterative methods. Furthermore, the solution obtained is in general only approximate. But in contrast to the general case, there is a straightforward possibility to construct a $\Phi$-function of two convex polytopes $T_{1}$ and $T_{2}$ which is based on properties of $\Phi$-functions.

This approach, considered in detail below, can be sketched as follows:

1. The polytope $T_{1}$ is considered to be fixed, i.e. $u_{1}=0$.

2. Facets of the polytope $T_{12}(0)$ are constructed, so that $\gamma_{12}$ is calculated.

3. Equations of all planes determined by these facets are constructed taking into account a suitable orientation of their normal vectors.

4. Based on the equations obtained in step 3, a collection of functions is formed which yields $\Phi\left(0, u_{2}\right)$. Finally, a $\Phi$-function $\Phi_{12}\left(u_{1}, u_{2}\right)$ is constructed.

3. Examples of $\Phi$-functions for $2 \mathrm{D}$ objects. In order to illustrate the strategy for obtaining $\Phi$-functions, we discuss three examples with simple geometric objects in $\mathbb{R}^{2}$. 
3.1. $\Phi$-functions of two circles. Let $C_{1}$ and $C_{2}$ be two circles of radii $r_{1}$ and $r_{2}$, respectively. When $C_{i}(i \in\{1,2\})$ is placed with center (pole) at $\left(x_{i}, y_{i}\right)$, then the notation $C_{i}\left(x_{i}, y_{i}\right)$ is used.

Without loss of generality, $\left(x_{1}, y_{1}\right)=(0,0)$ is assumed during the construction. Then it is evident that if the pole $\left(x_{2}, y_{2}\right)$ of $C_{2}$ moves along the curve

$$
\varphi\left(x_{2}, y_{2}\right)=0, \quad \text { where } \varphi(x, y)=\left(r_{1}+r_{2}\right)^{2}-x^{2}-y^{2},
$$

then the circles $C_{1}(0,0)$ and $C_{2}\left(x_{2}, y_{2}\right)$ are tangent. This means that the equation $\varphi\left(x_{2}, y_{2}\right)=0$ can be taken to describe the 0 -level surface of a $\Phi$ function of two circles. Obviously, the function $\varphi(x, y)$ is everywhere defined in $\mathbb{R}^{2}$. Therefore, to extend it onto the whole space $\mathbb{R}^{2}$ to obtain a $\Phi$-function, it is sufficient to choose an appropriate orientation of $\varphi(x, y)$. It is easy to verify that if the placement parameters $\left(x_{2}, y_{2}\right)$ are such that $C_{1}(0,0) \cap$ $C_{2}\left(x_{2}, y_{2}\right)=\emptyset$ then $-\varphi\left(x_{2}, y_{2}\right)>0$, and if int $C_{1}(0,0) \cap \operatorname{int} C_{2}\left(x_{2}, y_{2}\right) \neq \emptyset$ then $-\varphi\left(x_{2}, y_{2}\right)<0$. Hence,

$$
\Phi_{12}\left(0,0, x_{2}, y_{2}\right):=-\varphi\left(x_{2}, y_{2}\right)=x_{2}^{2}+y_{2}^{2}-\left(r_{1}+r_{2}\right)^{2}
$$

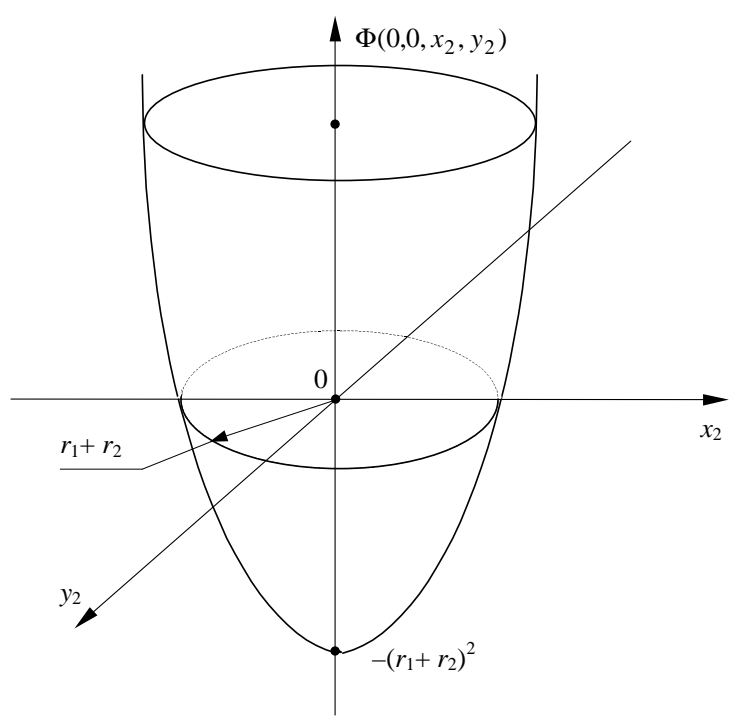

Fig. 1. $\Phi$-function of two circles

describes a paraboloid of rotation with vertex $\left(0,0,-\left(r_{1}+r_{2}\right)^{2}\right)$ (Fig. 1). By general properties of $\Phi$-functions, a $\Phi$-function of two circles $C_{1}$ and $C_{2}$ is as follows:

$$
\Phi_{12}\left(u_{1}, u_{2}\right)=\left(x_{2}-x_{1}\right)^{2}+\left(y_{2}-y_{1}\right)^{2}-\left(r_{1}+r_{2}\right)^{2} .
$$


Another possibility is to take

$$
\widetilde{\varphi}(x, y)=r_{1}+r_{2}-\sqrt{x^{2}+y^{2}} .
$$

It is easy to see that the equations $\varphi(x, y)=0$ and $\widetilde{\varphi}(x, y)=0$ define the same circle of radius $r_{1}+r_{2}$ with center at $(0,0)$. Therefore $\widetilde{\varphi}$ can also be used to construct a $\Phi$-function. Regarding an appropriate orientation of $\widetilde{\varphi}$ one obtains

$$
\widetilde{\Phi}_{12}\left(x_{1}, y_{1}, x_{2}, y_{2}\right)=\sqrt{\left(x_{2}-x_{1}\right)^{2}+\left(y_{2}-y_{1}\right)^{2}}-r_{1}-r_{2} .
$$

$\widetilde{\Phi}_{12}\left(0,0, x_{2}, y_{2}\right)$ describes a cone with vertex $\left(0,0,-\left(r_{1}+r_{2}\right)\right)$ (Fig. 2$)$.

The functions $\Phi_{12}$ and $\widetilde{\Phi}_{12}$ differ for example in their gradients:

$$
\left\|\nabla \Phi_{12}\right\|=2 \sqrt{2} \cdot \sqrt{\left(x_{2}-x_{1}\right)^{2}+\left(y_{2}-y_{1}\right)^{2}}, \quad\left\|\nabla \widetilde{\Phi}_{12}\right\|=\sqrt{2} .
$$

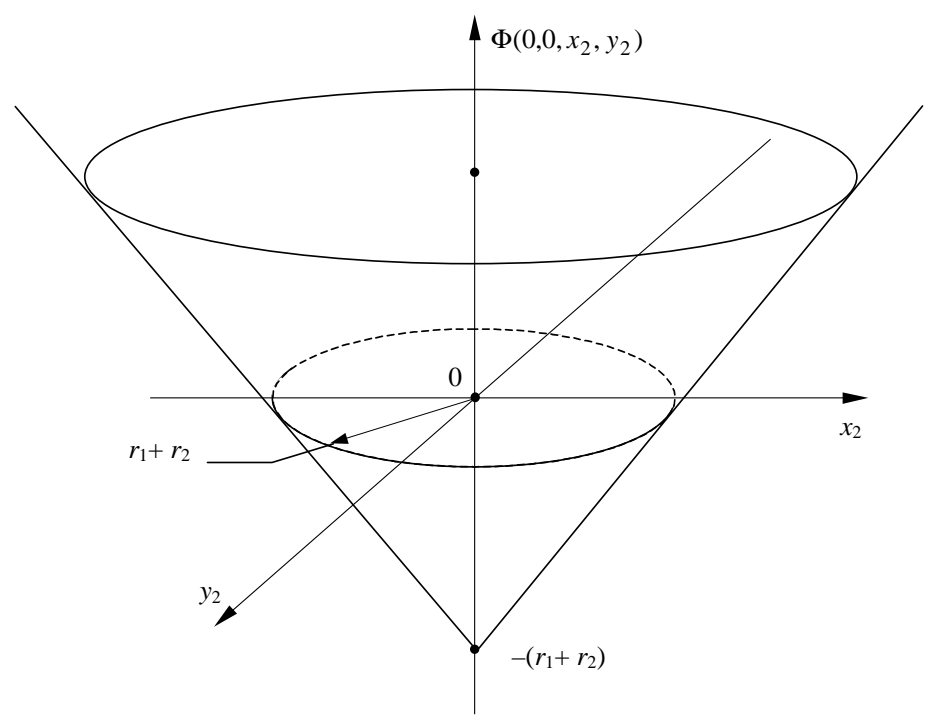

Fig. 2. Normalized $\Phi$-function of two circles

The value $\widetilde{\Phi}_{12}\left(x_{1}, y_{1}, x_{2}, y_{2}\right)$ is equal to the distance between $C_{1}\left(x_{1}, y_{1}\right)$ and $C_{2}\left(x_{2}, y_{2}\right)$ if $C_{1}\left(x_{1}, y_{1}\right) \cap C_{2}\left(x_{2}, y_{2}\right)=\emptyset$. Thus, this function is a normalized $\Phi$-function according to Definition 2.

Furthermore, $\min \Phi_{12}\left(x_{1}, y_{1}, x_{2}, y_{2}\right)=-\left(r_{1}+r_{2}\right)^{2}$ and $\min \widetilde{\Phi}_{12}\left(x_{1}, y_{1}\right.$, $\left.x_{2}, y_{2}\right)=-\left(r_{1}+r_{2}\right)$, i.e. the "measure" of maximal overlap of $C_{1}\left(x_{1}, y_{1}\right)$ and $C_{2}\left(x_{2}, y_{2}\right)$ is $-\left(r_{1}+r_{2}\right)^{2}$ or $-\left(r_{1}+r_{2}\right)$, respectively.

3.2. $\Phi$-function of two rectangles. Let $R_{1}$ and $R_{2}$ be rectangles with sides $2 a_{1}, 2 b_{1}$ and $2 a_{2}, 2 b_{2}$, respectively. It is assumed that their poles coincide with their symmetry centers. Again the placement parameters are denoted 
by $\left(x_{1}, y_{1}\right)$ and $\left(x_{2}, y_{2}\right)$, respectively. As above, $\left(x_{1}, y_{1}\right)=(0,0)$ is considered first. It is known [13], [8] that if the pole $\left(x_{2}, y_{2}\right)$ of $R_{2}$ moves along the frontier of the rectangle $R_{12}$ with vertices $(A, B),(-A, B),(-A,-B)$, $(A,-B)$, where $A=a_{1}+a_{2}, B=b_{1}+b_{2}$, then the rectangles $R_{1}(0,0)$ and $R_{2}\left(x_{2}, y_{2}\right)$ are tangent. Hence,

$$
R_{12}=\left\{(x, y) \in \mathbb{R}^{2}:-A \leq x \leq A,-B \leq y \leq B\right\}, \quad \gamma_{12}=\text { fr } R_{12} .
$$

This means that fr $R_{12}$ is the 0-level surface of $\Phi_{12}\left(0,0, x_{2}, y_{2}\right)$ defined for $R_{1}(0,0)$ and $R_{2}\left(x_{2}, y_{2}\right)$. Hence, a representation of fr $R_{12}$ by means of equations is needed. Let

$$
\begin{array}{ll}
\psi_{1}(x, y)=x-A, & \psi_{2}(x, y)=y-B \\
\psi_{3}(x, y)=-x-A, & \psi_{4}(x, y)=-y-B .
\end{array}
$$

Then

$$
R_{12}=\left\{(x, y) \in \mathbb{R}^{2}: \psi_{i}(x, y) \leq 0, i=1, \ldots, 4\right\}
$$

and hence fr $R_{12}=\left\{(x, y) \in R_{12}: \psi(x, y)=0\right\}$, where

$$
\psi(x, y):=\max \left\{\psi_{1}(x, y), \psi_{2}(x, y), \psi_{3}(x, y), \psi_{4}(x, y)\right\} .
$$

In these definitions an appropriate orientation is obtained if

$$
\psi\left(x_{2}, y_{2}\right)<0 \quad \text { if } \operatorname{int} R_{1}(0,0) \cap \operatorname{int} R_{2}\left(x_{2}, y_{2}\right) \neq \emptyset .
$$

Since additionally

$$
\psi\left(x_{2}, y_{2}\right)>0 \quad \text { if } R_{1}(0,0) \cap R_{2}\left(x_{2}, y_{2}\right)=\emptyset,
$$

a $\Phi$-function is obtained as follows:

$$
\Phi_{12}\left(x_{1}, y_{1}, x_{2}, y_{2}\right):=\psi\left(x_{2}-x_{1}, y_{2}-y_{1}\right) .
$$

Note that $\min \Phi_{12}\left(x_{1}, y_{1}, x_{2}, y_{2}\right)=\max \{-A,-B\}$. Thus, if $A=B$ then $\Phi_{12}\left(0,0, x_{2}, y_{2}\right)$ describes an inverted pyramid with vertex $(0,0,-A)$. This means that the maximal "intersection measure" of $R_{1}\left(x_{1}, y_{1}\right)$ and $R_{2}\left(x_{2}, y_{2}\right)$ is $-A$. (In the case of $A \neq B$, instead of the vertex an edge occurs.)

It should be noted that $\Phi_{12}$ is not normalized since $\Phi_{12}\left(u_{1}, u_{2}\right)$ is not equal to the distance between $R_{1}\left(u_{1}\right)$ and $R_{2}\left(u_{2}\right)$ in the general case. This happens when $\left(u_{1}, u_{2}\right)$ satisfy one of the following systems:

$$
\begin{aligned}
& \left\{\begin{array} { l } 
{ \psi _ { 1 } ( u _ { 2 } - u _ { 1 } ) > 0 , } \\
{ \psi _ { 4 } ( u _ { 2 } - u _ { 1 } ) > 0 , }
\end{array} \quad \left\{\begin{array}{l}
\psi_{1}\left(u_{2}-u_{1}\right)>0, \\
\psi_{2}\left(u_{2}-u_{1}\right)>0,
\end{array}\right.\right. \\
& \left\{\begin{array} { l } 
{ \psi _ { 2 } ( u _ { 2 } - u _ { 1 } ) > 0 , } \\
{ \psi _ { 3 } ( u _ { 2 } - u _ { 1 } ) > 0 , }
\end{array} \quad \left\{\begin{array}{l}
\psi_{4}\left(u_{2}-u_{1}\right)>0, \\
\psi_{3}\left(u_{2}-u_{1}\right)>0 .
\end{array}\right.\right.
\end{aligned}
$$

A normalized $\Phi$-function of two rectangles $R_{1}$ and $R_{2}$ is considered in the next subsection as a particular case of a $\Phi$-function of a rectangle and a circle. 
3.3. $\Phi$-function of a rectangle and a circle. Let $R$ be a rectangle with sides $2 a$ and $2 b$, and let $C$ be a circle of radius $r$. The poles of $R$ and $C$ are at their symmetry centers. The placement parameters of $R$ and $C$ are denoted by $\left(x_{1}, y_{1}\right)$ and $\left(x_{2}, y_{2}\right)$, respectively. Again, in order to construct a $\Phi$-function, $\left(x_{1}, y_{1}\right)=(0,0)$ is assumed.

Let $A=a+r, B=b+r$. It is easy to see that the rectangle and the circle are tangent if the pole $\left(x_{2}, y_{2}\right)$ of $C$ moves along the following curve which consists of straight-line segments and circular arcs alternately:

$$
\text { line }((A,-b),(A, b)), \quad \operatorname{arc}((A, b),(a, B)), \quad \operatorname{line}((a, B),(-a, B)),
$$

$\operatorname{arc}((-a, B),(-A, b)), \quad \operatorname{line}((-A, b),(-A,-b)), \quad \operatorname{arc}((-A,-b),(-a,-B))$, line $((-a,-B),(a,-B)), \quad \operatorname{arc}((a,-B),(A,-b))$.

Let

$$
\begin{array}{ll}
\psi_{1}(x, y)=x-A, & \psi_{2}(x, y)=y-B \\
\psi_{3}(x, y)=-x-A, & \psi_{4}(x, y)=-y-B
\end{array}
$$

and let

$$
\begin{array}{ll}
\varphi_{1}(x, y)=\sqrt{(x-a)^{2}+(y-b)^{2}}-r, & \varphi_{2}(x, y)=\sqrt{(x+a)^{2}+(y-b)^{2}}-r, \\
\varphi_{3}(x, y)=\sqrt{(x+a)^{2}+(y+b)^{2}}-r, & \varphi_{4}(x, y)=\sqrt{(x-a)^{2}+(y+b)^{2}}-r .
\end{array}
$$

The corresponding $\Phi$-function of $R(0,0)$ and $C\left(x_{2}, y_{2}\right)$ is

$$
\Phi_{12}\left(0,0, x_{2}, y_{2}\right):= \begin{cases}\varphi_{1}\left(x_{2}, y_{2}\right) & \text { if } x_{2} \geq a, y_{2} \geq b, \\ \varphi_{2}\left(x_{2}, y_{2}\right) & \text { if } x_{2} \leq-a, y_{2} \geq b, \\ \varphi_{3}\left(x_{2}, y_{2}\right) & \text { if } x_{2} \leq-a, y_{2} \leq-b, \\ \varphi_{4}\left(x_{2}, y_{2}\right) & \text { if } x_{2} \geq a, y_{2} \leq-b, \\ \varphi\left(x_{2}, y_{2}\right) & \text { otherwise, }\end{cases}
$$

where $\varphi(x, y):=\max \left\{\psi_{1}(x, y), \psi_{2}(x, y), \psi_{3}(x, y), \psi_{4}(x, y)\right\}$.

However, such a representation of this $\Phi$-function $\Phi_{12}\left(0,0, x_{2}, y_{2}\right)$ may be unsuitable when a feasible region of an optimization placement problem has to be described since any word information must be excluded. Therefore we give another representation of $\Phi_{12}\left(0,0, x_{2}, y_{2}\right)$. In order to obtain it, consider the first quadrant, i.e. $x \geq 0$ and $y \geq 0$. If $x>a$ and $y>b$ then $\min \left\{\psi_{1}(x, y), \psi_{2}(x, y)\right\}<\varphi_{1}(x, y)$ but $\varphi_{1}(x, y)$ gives the distance. Therefore an additional function $\psi_{12}(x, y)=x+y-a-b-r$ is used. Since $\psi_{12}(x, y) \geq \varphi_{1}(x, y)$ for $x \geq a$ and $y \geq b, \psi_{12}(x, y) \leq \psi_{1}(x, y)$ for $x \geq a$ and $-b \leq y \leq b$, and $\psi_{12}(x, y) \leq \psi_{2}(x, y)$ for $-a \leq x \leq a$ and $y \geq b$, we see that for $x \geq 0$ and $y \geq 0, \Phi_{12}\left(0,0, x_{2}, y_{2}\right)$ can be represented by $\min \left\{\varphi_{1}\left(x_{2}, y_{2}\right), \max \left\{\psi_{1}\left(x_{2}, y_{2}\right), \psi_{2}\left(x_{2}, y_{2}\right), \psi_{12}\left(x_{2}, y_{2}\right)\right\}\right\}$.

Summarizing, the following representation results:

$$
\begin{aligned}
& \Phi_{12}\left(0,0, x_{2}, y_{2}\right) \\
& \quad=\min \left\{\varphi_{0}\left(x_{2}, y_{2}\right), \varphi_{1}\left(x_{2}, y_{2}\right), \varphi_{2}\left(x_{2}, y_{2}\right), \varphi_{3}\left(x_{2}, y_{2}\right), \varphi_{4}\left(x_{2}, y_{2}\right)\right\}
\end{aligned}
$$


where

$$
\varphi_{0}(x, y):=\max \left\{\varphi(x, y), \psi_{12}(x, y), \psi_{23}(x, y), \psi_{34}(x, y), \psi_{41}(x, y)\right\}
$$

and $\psi_{12}(x, y)=x+y-c, \psi_{23}(x, y)=-x+y-c, \psi_{34}(x, y)=-x-y-$ $c, \psi_{41}(x, y)=x-y-c$ with $c:=a+b+r$. Hence, a normalized $\Phi$-function of a rectangle and a circle is as follows:

$$
\Phi_{12}\left(u_{1}, u_{2}\right)=\min _{i=0, \ldots, 4} \varphi_{i}\left(x_{2}-x_{1}, y_{2}-y_{1}\right) .
$$

In order to construct a normalized $\Phi$-function of two rectangles $R_{1}$ and $R_{2}$ (as considered in Example 2) we now only have to define $r, a$ and $b$ in a suitable manner. If

$$
r:=0, \quad a:=a_{1}+a_{2} \quad b:=b_{1}+b_{2}
$$

then $\Phi_{12}$ defined in (6) or (7) is a normalized $\Phi$-function of $R_{1}$ and $R_{2}$.

4. 0-level surface of a $\Phi$-function for convex polytopes. In the following, three-dimensional objects will be considered. The description is restricted to the case of (full-dimensional) convex closed polytopes $T$, i.e. $\operatorname{int}(T) \neq \emptyset$ and $T=\operatorname{cl}(\operatorname{int}(T))$. In this section, one of the possibilities to construct the 0 -level surface of a $\Phi$-function of two polytopes $T_{1}$ and $T_{2}$ is discussed.

An arbitrary closed polytope in $\mathbb{R}^{3}$ can be described in various ways (see for example [3], [7], [5], [6]). In particular, any convex polytope is entirely determined by its vertices. In many cases, a convex polytope is considered as the intersection of a finite number of half spaces. A polytope can also be described by its facets (two-dimensional faces). The latter variant seems to be most suitable for the case when nonconvex polytopes have to be regarded. So we mainly use a facet-oriented characterization of polytopes.

Let $v_{j}^{k}=\left(x_{j}^{k}, y_{j}^{k}, z_{j}^{k}\right), j=1, \ldots, M_{k}$, denote the vertices of the polytope $T_{k}, k=1,2$. Since $T_{k}$ is assumed to be convex there exists a representation by means of a minimal number $N_{k}$ of half spaces $H_{i}^{k}$. These half spaces are determined by the facets of $T_{k}$. Let

$$
h_{i}^{k}\left\langle v_{i 1}^{k}, v_{i 2}^{k}, \ldots, v_{i m_{i}}^{k}\right\rangle=\operatorname{conv}\left\{v_{i 1}^{k}, v_{i 2}^{k}, \ldots, v_{i m_{i}}^{k}\right\}
$$

denote the facet which is determined by the $m_{i}\left(m_{i} \geq 3\right)$ vertices $v_{i p}^{k} \in V_{k}=$ $\left\{v_{j}^{k}: j=1, \ldots, M_{k}\right\}, p=1, \ldots, m_{i}$. ("conv" denotes the convex hull of a set.) To any facet $h_{i}^{k}, i=1, \ldots, N_{k}$, there corresponds a plane

where

$$
H_{i}^{k}:=\left\{v=(x, y, z) \in \mathbb{R}^{3}: f_{i}^{k}(v)=f_{i}^{k}(x, y, z)=0\right\}
$$

$$
f_{i}^{k}(v)=f_{i}^{k}(x, y, z)=A_{i}^{k} x+B_{i}^{k} y+C_{i}^{k} z+D_{i}^{k}=0
$$

is determined by $f_{i}^{k}\left(v_{i p}^{k}\right)=0, p=1, \ldots, m_{i}$. Regarding an appropriate orientation of $f_{i}^{k}$ so that $f_{i}^{k}\left(v_{j p}^{k}\right) \leq 0$ for all $p$ and $j$, a (minimal) representation of $T_{k}$ by means of half spaces is as follows: 


$$
T_{k}:=\left\{(x, y, z) \in \mathbb{R}^{3}: f_{i}^{k}(x, y, z) \leq 0, i=1, \ldots, N_{k}\right\} .
$$

In addition, without loss of generality, it is assumed that the vertices $v_{i 1}^{k}, v_{i 2}^{k}, \ldots, v_{i m_{i}}^{k}$ of each facet form a counterclockwise circuit when looking on $T_{k}$ from the outside.

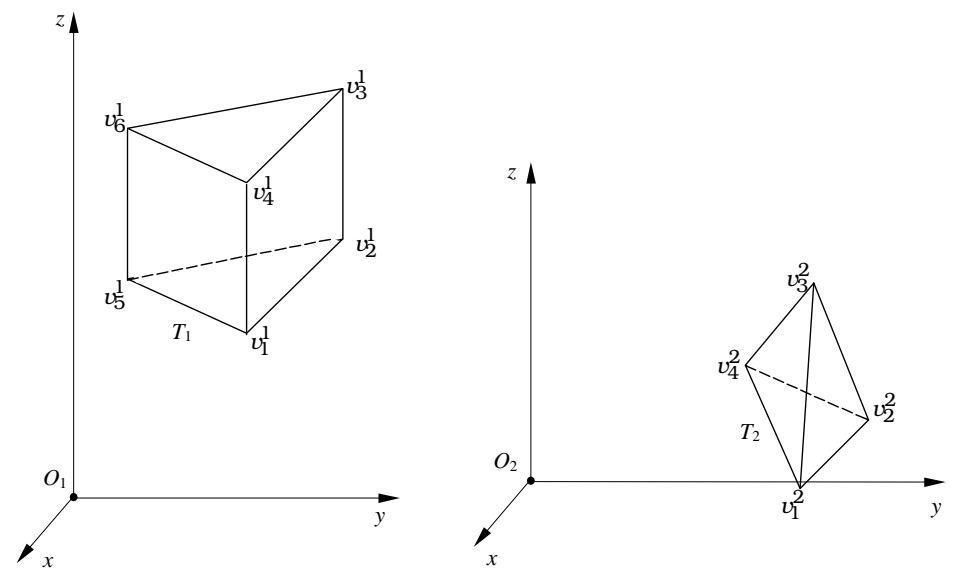

Fig. 3. Example

Table 1. Vertex coordinates of $T_{1}$ and $T_{2}$

\begin{tabular}{r|rrrrrr||c|rrrr}
\hline$T_{1}$ & $v_{1}^{1}$ & $v_{2}^{1}$ & $v_{3}^{1}$ & $v_{4}^{1}$ & $v_{5}^{1}$ & $v_{6}^{1}$ & $T_{2}$ & $v_{1}^{2}$ & $v_{2}^{2}$ & $v_{3}^{2}$ & $v_{4}^{2}$ \\
\hline$x$ & 4.0 & 0.0 & 0.0 & 4.0 & 2.0 & 2.0 & $x$ & 5.0 & 2.0 & 3.0 & 0.0 \\
$y$ & 10.0 & 10.0 & 10.0 & 10.0 & 4.0 & 4.0 & $y$ & 15.0 & 15.0 & 14.0 & 9.0 \\
$z$ & 11.0 & 11.0 & 19.0 & 19.0 & 11.0 & 19.0 & $z$ & 4.0 & 4.0 & 10.0 & 4.0 \\
\hline
\end{tabular}

In order to illustrate the notations, consider the example depicted in Fig. 3. The vertex coordinates of $T_{1}$ and $T_{2}$ are given in Table 1 . The facets $h_{i}^{k}, i=1, \ldots, N_{k}$, of $T_{k}, k=1,2$, are specified by appropriate sequences of vertices:

$$
\begin{aligned}
h_{1}^{1}\left\langle v_{11}^{1}, v_{12}^{1}, v_{13}^{1}, v_{14}^{1}\right\rangle & =h_{1}^{1}\left\langle v_{1}^{1}, v_{2}^{1}, v_{3}^{1}, v_{4}^{1}\right\rangle, \\
h_{2}^{1}\left\langle v_{21}^{1}, v_{22}^{1}, v_{23}^{1}, v_{24}^{1}\right\rangle & =h_{2}^{1}\left\langle v_{1}^{1}, v_{4}^{1}, v_{6}^{1}, v_{5}^{1}\right\rangle, \\
h_{3}^{1}\left\langle v_{31}^{1}, v_{32}^{1}, v_{33}^{1}, v_{34}^{1}\right\rangle & =h_{3}^{1}\left\langle v_{2}^{1}, v_{5}^{1}, v_{6}^{1}, v_{3}^{1}\right\rangle, \\
h_{4}^{1}\left\langle v_{41}^{1}, v_{42}^{1}, v_{43}^{1}\right\rangle & =h_{4}^{1}\left\langle v_{3}^{1}, v_{6}^{1}, v_{4}^{1}\right\rangle, \\
h_{5}^{1}\left\langle v_{51}^{1}, v_{52}^{1}, v_{53}^{1}\right\rangle & =h_{5}^{1}\left\langle v_{1}^{1}, v_{5}^{1}, v_{2}^{1}\right\rangle, \\
h_{1}^{2}\left\langle v_{11}^{2}, v_{12}^{2}, v_{13}^{2}\right\rangle & =h_{1}^{2}\left\langle v_{1}^{2}, v_{2}^{2}, v_{3}^{2}\right\rangle, \\
h_{2}^{2}\left\langle v_{21}^{2}, v_{22}^{2}, v_{23}^{2}\right\rangle & =h_{2}^{2}\left\langle v_{1}^{2}, v_{3}^{2}, v_{4}^{2}\right\rangle, \\
h_{3}^{2}\left\langle v_{31}^{2}, v_{32}^{2}, v_{33}^{2}\right\rangle & =h_{3}^{2}\left\langle v_{2}^{2}, v_{4}^{2}, v_{3}^{2}\right\rangle, \\
h_{4}^{2}\left\langle v_{41}^{2}, v_{42}^{2}, v_{43}^{2}\right\rangle & =h_{4}^{2}\left\langle v_{1}^{2}, v_{4}^{2}, v_{2}^{2}\right\rangle .
\end{aligned}
$$


Note that, in contrast to the approach described below, there also exist some possibilities to construct a $\Phi$-function by means of translated planes (half space representation). But in the latter case, no facet representation of the 0 -level surface of a $\Phi$-function is obtained and therefore it has to be computed in another way.

In order to construct facets of the 0-level surface of a $\Phi$-function, we assume that $T_{1}$ is fixed with $u_{1}=\left(x_{1}, y_{1}, z_{1}\right)=(0,0,0)$. Due to the properties of $\Phi$-functions mentioned in Section 2, the equation $\Phi\left(0,0,0, x_{2}, y_{2}, z_{2}\right)=0$ describes the frontier of the convex polytope

$$
T_{12}:=\left\{u \in \mathbb{R}^{3}: T_{1} \cap T_{2}(u) \neq \emptyset\right\}=T_{1}+(-1) T_{2}(0) .
$$

Let $\gamma_{12}:=\operatorname{fr}\left(T_{12}\right)$. By definition, if $u_{2}=\left(x_{2}, y_{2}, z_{2}\right) \in \gamma_{12}$ then $T_{1}(0)$ and $T_{2}\left(u_{2}\right)$ are in contact or tangent, i.e. $\operatorname{int} T_{1} \cap \operatorname{int} T_{2}\left(u_{2}\right)=\emptyset$ and $\operatorname{fr}\left(T_{1}\right) \cap$ $\operatorname{fr}\left(T_{2}\left(u_{2}\right)\right) \neq \emptyset$. This implies that for any $u_{2} \in \gamma_{12}$ there exist $t^{*} \in \operatorname{fr} T_{1}(0)$ and $t^{* *} \in$ fr $T_{2}(0)$ such that $t^{*}=u_{2}+t^{* *}$. On the other hand, if $T_{1}(0)$ and $T_{2}\left(u_{2}\right)$ are in contact (tangent) then $u_{2} \in \gamma_{12}$ and $u_{2}=t^{*}-t^{* *}$.

In order to construct $\gamma_{12}$ for two convex polytopes only the following six situations to be in contact are of interest. In these cases (moving $T_{2}$ along the frontier of $T_{1}$, or vice versa) two-dimensional faces of $T_{12}=\operatorname{conv}\left(\gamma_{12}\right)$ arise:

- a vertex or an edge or a facet of $T_{2}$ is in contact with a facet of $T_{1}$,

- a vertex or an edge of $T_{1}$ is in contact with a facet of $T_{2}$, or

- an edge of $T_{2}$ is in contact with an edge of $T_{1}$.

The remaining situations do not lead to (two-dimensional) facets of $T_{12}$ and of $\gamma_{12}$.

The approach given below is to successively generate all facets of the surface $\gamma_{12}$ and to define half spaces whose intersection is $T_{12}$. In more detail, under certain conditions any kind of contact may lead to a facet of $\gamma_{12}$. Taking into account that $T_{2}$ is movable and $T_{1}$ is fixed, we consider successively all the six situations and discuss the rules for constructing facets of $\gamma_{12}$. Furthermore we formulate sufficient conditions for the resulting face to be a facet of $\gamma_{12}$.

1. A vertex of $T_{2}$ is in contact with a facet of $T_{1}$. More precisely, let $h_{i}^{1}\left\langle v_{i 1}^{1}, v_{i 2}^{1}, \ldots, v_{i m_{i}}^{1}\right\rangle$ be some facet of $T_{1}$. The plane $H_{i}^{1}=\{(x, y, z)$ : $\left.f_{i}^{1}(x, y, z)=0\right\}$ determined by $h_{i}^{1}$ is a separating plane of $T_{1}(0)$ and $T_{2}(u)$ for any $u \in \mathbb{R}^{3}$ with

$$
\min \left\{f_{i}^{1}(v+u): v \in T_{2}(u)\right\} \geq 0 .
$$

Since $T_{2}$ is assumed to be convex, $H_{i}^{1}$ is a separating plane of $T_{1}(0)$ and $T_{2}(u)$ for any $u \in \mathbb{R}^{3}$ with

$$
\min \left\{f_{i}^{1}\left(v_{j}^{2}+u\right): j=1, \ldots, M_{2}\right\} \geq 0 .
$$


Let

$$
J_{i}^{2}:=\left\{j^{\prime} \in\left\{1, \ldots, M_{2}\right\}: f_{i}^{1}\left(v_{j^{\prime}}^{2}\right)=\min \left\{f_{i}^{1}\left(v_{j}^{2}\right): j=1, \ldots, M_{2}\right\}\right\} .
$$

Then the facet $h_{i}^{1}\left\langle v_{i 1}^{1}, v_{i 2}^{1}, \ldots, v_{i m_{i}}^{1}\right\rangle$ determines a facet of $\gamma_{12}$ congruent to $h_{i}^{1}$ if $\operatorname{card}\left(J_{i}^{2}\right)=1$. Let $J_{i}^{2}=\{\alpha\}$. Then exactly one point of $T_{2}(u)$ may be in contact with $T_{1}$ in case $H_{i}^{1}$ is a separating plane. The set of translation vectors $u$ of $T_{2}$ is given by

$$
\left\{u \in \mathbb{R}^{3}: u=u^{\prime}-v_{\alpha}^{2}, u^{\prime} \in H_{i}^{1} \cap T_{1}(0)\right\} .
$$

Hence, the corresponding facet of $\gamma_{12}$, denoted by $q$, is

$$
q=\left\langle v_{i 1}^{1}-v_{\alpha}^{2}, v_{i 2}^{1}-v_{\alpha}^{2}, \ldots, v_{i m_{i}}^{1}-v_{\alpha}^{2}\right\rangle .
$$

2. An edge of $T_{2}$ is in contact with a facet of $T_{1}$. This situation occurs when $\operatorname{card}\left(J_{i}^{2}\right)=2$. Let $J_{i}^{2}=\{\alpha, \beta\}$, i.e. $v_{\alpha}^{2}$ and $v_{\beta}^{2}$ define an edge

$$
e^{2}:=\left\{v: v=\lambda v_{\alpha}^{2}+(1-\lambda) v_{\beta}^{2}, \lambda \in[0,1]\right\}
$$

of $T_{2}(0)$. It is known that the edge $e^{2}$ and the facet $h_{i}^{1}$ determine a facet $q$ of $T_{12}$ as follows:

$$
q=h_{i}^{1}+(-1) e^{2}:=\left\{v^{1}-v^{2}: v^{1} \in h_{i}^{1}, v^{2} \in e^{2}\right\} .
$$

Since $q$ is the Minkowski sum of $h_{i}^{1}$ and $-e^{2}$, the vertices of $q$ are in the set $Q=\left\{v_{i j}^{1}-v_{\alpha}^{2}, v_{i j}^{1}-v_{\beta}^{2}: j=1, \ldots, m_{i}\right\}$. In order to identify points in $Q$ which are vertices of $q$, algorithms which work in two-dimensional space can be used. Therefore we project $h_{i}^{1}$ and $e^{2}$ onto a suitable coordinate plane, e.g., the $x y$-plane, obtaining a polygon $\operatorname{pr}_{x y}\left(h_{i}^{1}\right)$ with vertices $\left(x_{i j}^{1}, y_{i j}^{1}\right), j=$ $1, \ldots, n_{i}$, and a closed interval $\operatorname{pr}_{x y}\left(e^{2}\right)$ with vertices $\left(x_{\alpha}^{2}, y_{\alpha}^{2}\right)$ and $\left(x_{\beta}^{2}, y_{\beta}^{2}\right)$. Using one of the algorithms to construct the 0-level surface of a $\Phi$-function in $\mathbb{R}^{2}$ (see below), we identify the vertices of the projection of $q$, and hence, the vertices of $q$. For example, if $\left(\bar{v}_{i j}^{1}\left(x_{i j}^{1}, y_{i j}^{1}\right), \bar{v}_{\alpha}^{2}\left(x_{\alpha}^{2}, y_{\alpha}^{2}\right)\right)$ is a vertex of the polygon $\operatorname{pr}_{x y}(q)$ then $\left(x_{i j}^{1}-x_{\alpha}^{2}, y_{i j}^{1}-y_{\alpha}^{2}, z_{i j}^{1}-z_{\alpha}^{2}\right)$ is a vertex of $q$.

The choice of the projection plane depends on the angle between $H_{i}^{1}$ and the coordinate axes (cf. below).

3. A facet of $T_{2}$ is in contact with a facet of $T_{1}$. This means that the facets lie in parallel planes. As a consequence of the first two situations, if $\operatorname{card}\left(J_{i}^{2}\right)=\chi \geq 3$ then a facet of $T_{2}$ may be in contact with $T_{1}$. Let $J_{i}^{2}=\left\{j_{\nu}: \nu=1, \ldots, \chi\right\}$. Since $v_{j_{\nu}}^{2}$ are vertices of $T_{2}$ there exists an index $s$ with $H_{s}^{2}$ parallel to $H_{i}^{1}$.

Similarly to the above, the facets $h_{s}^{2}$ and $h_{i}^{1}$ determine a facet $q$ of $T_{12}$ as follows:

$$
q=h_{i}^{1}+(-1) h_{s}^{2}:=\left\{v^{1}-v^{2}: v^{1} \in h_{i}^{1}, v^{2} \in h_{s}^{2}\right\} .
$$

Now, $q$ is the Minkowski sum of $h_{i}^{1}$ and $-h_{s}^{2}$. Hence the vertices of $q$ are in $Q=\left\{v_{i j}^{1}-v_{j_{\nu}}^{2}: j=1, \ldots, m_{i}, \nu=1, \ldots, \chi\right\}$. 
Again, using a suitable projection and two-dimensional algorithms, we can calculate the vertices of the projection of $q$, and hence also for $q$ (see below).

4. A vertex of $T_{1}$ is in contact with a facet of $T_{2}$ and 5 . An edge of $T_{1}$ is in contact with a facet of $T_{2}$. These two cases can be handled similarly to cases 1 and 2. Let $h_{s}^{2}\left\langle v_{s 1}^{2}, v_{s 2}^{2}, \ldots, v_{s m_{s}}^{2}\right\rangle$ be any facet of $T_{2}$. Set

$$
J_{s}^{1}:=\left\{j^{\prime} \in\left\{1, \ldots, M_{1}\right\}: f_{s}^{2}\left(v_{j^{\prime}}^{1}\right)=\min \left\{f_{s}^{2}\left(v_{j}^{1}\right): j=1, \ldots, M_{1}\right\}\right\} .
$$

If $\operatorname{card}\left(J_{s}^{1}\right)=1$ then case 4 holds, and if $\operatorname{card}\left(J_{s}^{1}\right)=2$ then case 5 occurs. In the case of $\operatorname{card}\left(J_{s}^{1}\right) \geq 3$ no new situation arises.

6. An edge of $T_{1}$ is in contact with an edge of $T_{2}$. Let $e^{2}$ be an edge of $T_{2}$ connecting vertices $v_{t}^{2}$ and $v_{h}^{2}$, i.e. $e^{2}=\left\{v: v=\lambda v_{t}^{2}+(1-\lambda) v_{h}^{2}, \lambda \in[0,1]\right\}$, and let $e^{1}$ be an edge of $T_{1}$ connecting vertices $v_{t}^{1}$ and $v_{h}^{1}$ (Fig. 4). These two edges determine a facet $q$ of $\gamma_{12}$ if and only if they are not parallel and the plane

$$
H=\left\{v: v=v_{t}^{1}+\lambda\left(v_{h}^{1}-v_{t}^{1}\right)+\varrho\left(v_{h}^{2}-v_{t}^{2}\right), \lambda, \varrho \in \mathbb{R}^{1}\right\}=:\{v: f(v)=0\}
$$

separates $T_{1}(0)$ and $T_{2}\left(u_{2}\right)$ with $u_{2}=v_{t}^{1}-v_{t}^{2}$, i.e. $f\left(v_{j}^{1}\right) \leq 0$ for $j=$ $1, \ldots, M_{1}$, and $0 \leq f\left(v_{j}^{2}+u_{2}\right)$ for $j=1, \ldots, M_{2}$, and $f\left(v_{j}^{1}\right)<0$ for $j \neq t, h$ and $f\left(v_{j}^{2}+u_{2}\right)>0$ for $j \neq t, h$.

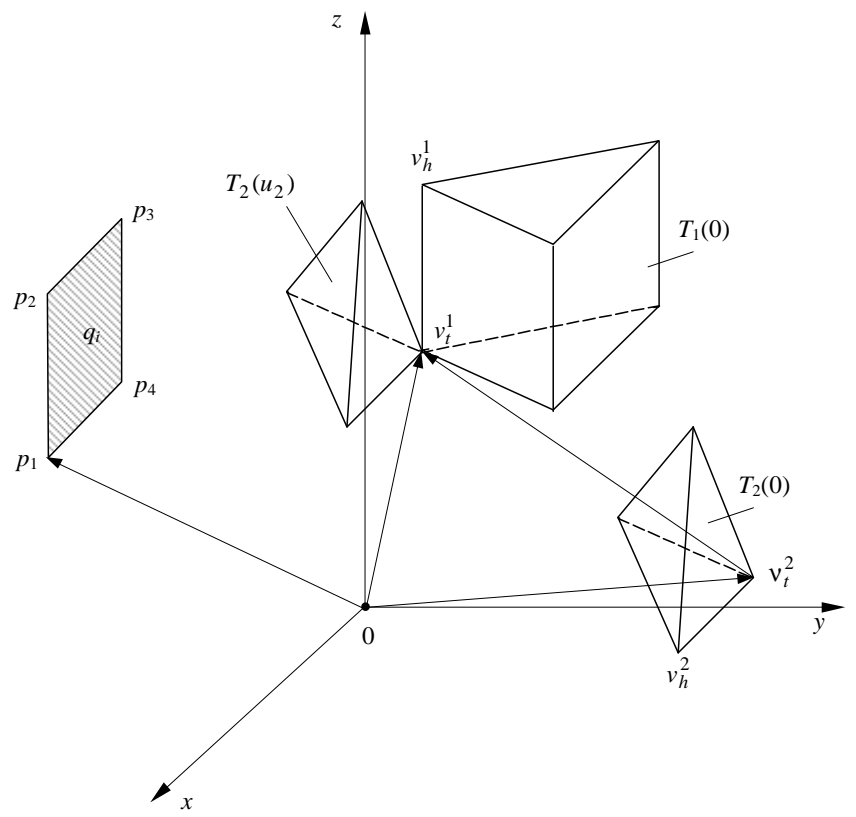

Fig. 4. Two edges form a facet 
The facet $q \subset \gamma_{12}$ determined by $e^{1}$ and $e^{2}$ may be represented as

$$
q=e^{1}+(-1) e^{2}=\left\{v^{1}-v^{2}: v^{1} \in e^{1}, v^{2} \in e^{2}\right\} .
$$

Hence, $q$ is a parallelogram parallel to $H$ with the vertices $p_{1}=v_{t}^{1}-v_{t}^{2}$, $p_{2}=v_{h}^{1}-v_{t}^{2}, p_{3}=v_{h}^{1}-v_{h}^{2}$, and $p_{4}=v_{t}^{1}-v_{h}^{2}$. Note that when calculating such a parallelogram one has to take care of numerical stability since for nearly parallel edges the resulting facet $q$ becomes very thin.

Summarizing, the facets of the surface $\gamma_{12}$ can be obtained as follows:

1. combine each facet of $T_{1}$ with an appropriate element (vertex, edge or facet) of $T_{2}$,

2. combine each facet of $T_{2}$ with an appropriate element (vertex or edge) of $T_{1}$, and plane.

3. consider any pair of edges of $T_{1}$ and $T_{2}$ which define a separating

The set of facets $q$ computed in this way describes the surface of $\gamma_{12}$.

5. $\Phi$-function of two convex polytopes. In the previous section, we have obtained all facets

$$
q_{i}=\left\langle v_{i 1}, \ldots, v_{i m_{i}}\right\rangle, \quad i=1, \ldots, N,
$$

of $\gamma_{12}$. Let $\left\{v_{j}: j \in J\right\}$ denote the set of vertices of $\gamma_{12}$. It remains to find the corresponding $\Phi$-function.

For that purpose, for each facet $q_{i}$ of $\gamma_{12}$, we construct a plane $H_{i}=\{u$ : $\left.f_{i}(u)=0\right\}$ determined by

$$
f_{i}(u)=f_{i}(x, y, z)=0 \quad \forall u \in q_{i} .
$$

The orientation of the plane is such that $f_{i}\left(v_{j}\right) \leq 0$ for all $j \in J$.

Obviously, $f_{i}$ can be calculated by solving the linear system

$$
f_{i}\left(v_{i j}\right)=0, \quad j=1, \ldots, m_{i},
$$

and choosing a suitable solution (with regard to orientation).

Since the polytopes $T_{1}$ and $T_{2}$ are convex, $T_{12}$ can be represented as

$$
\begin{aligned}
T_{12} & =\left\{u \in \mathbb{R}^{3}: f_{i}(u) \leq 0, i=1, \ldots, N\right\} \\
& =\left\{u \in \mathbb{R}^{3}: \max \left\{f_{i}(u): i=1, \ldots, N\right\} \leq 0\right\} .
\end{aligned}
$$

Hence, if $T_{2}$ is placed at $u_{2}$ and $u_{2} \in T_{12}$ then $T_{1}(0)$ and $T_{2}\left(u_{2}\right)$ are in contact or they overlap. Consequently,

$$
\Phi_{12}\left(0, u_{2}\right)=\max \left\{f_{1}\left(u_{2}\right), f_{2}\left(u_{2}\right), \ldots, f_{N}\left(u_{2}\right)\right\} .
$$

Therefore,

$$
\Phi_{12}\left(u_{1}, u_{2}\right)=\max \left\{f_{1}\left(u_{2}-u_{1}\right), f_{2}\left(u_{2}-y_{1}\right), \ldots, f_{N}\left(u_{2}-u_{1}\right)\right\}
$$

is a $\Phi$-function of the two polytopes $T_{1}\left(u_{1}\right)$ and $T_{2}\left(u_{2}\right)$. 
Example (cont.). For the polytopes $T_{1}$ and $T_{2}$ given in Table 1 (Fig. 3 ), the following 0 -level surface $\gamma_{12}$ results, characterized by its 12 facets:

$$
\begin{gathered}
q_{1}\left\langle v_{1}, v_{2}, v_{3}, v_{4}\right\rangle, q_{2}\left\langle v_{5}, v_{6}, v_{1}, v_{4}\right\rangle, q_{3}\left\langle v_{7}, v_{8}, v_{9}, v_{10}\right\rangle, q_{4}\left\langle v_{10}, v_{9}, v_{5}, v_{4}, v_{3}\right\rangle, \\
q_{5}\left\langle v_{11}, v_{12}, v_{13}\right\rangle, q_{6}\left\langle v_{12}, v_{6}, v_{8}\right\rangle, q_{7}\left\langle v_{2}, v_{13}, v_{7}\right\rangle, q_{8}\left\langle v_{1}, v_{6}, v_{12}, v_{11}\right\rangle, \\
q_{9}\left\langle v_{11}, v_{13}, v_{2}, v_{1}\right\rangle, q_{10}\left\langle v_{2}, v_{7}, v_{10}, v_{3}\right\rangle, q_{11}\left\langle v_{9}, v_{8}, v_{6}, v_{5}\right\rangle, q_{12}\left\langle v_{13}, v_{12}, v_{8}, v_{7}\right\rangle,
\end{gathered}
$$

where the coordinates of the vertices of $\gamma_{12}$ are given in Table 2. $\gamma_{12}$ is depicted in Fig. 5.

Table 2. Vertices of $\gamma_{12}$

\begin{tabular}{r|rrrrrrrrrrrrr}
\hline$\gamma$ & $v_{1}$ & $v_{2}$ & $v_{3}$ & $v_{4}$ & \multicolumn{1}{c}{$v_{5}$} & \multicolumn{1}{c}{$v_{6}$} & \multicolumn{1}{c}{$v_{7}$} & \multicolumn{1}{c}{$v_{8}$} & $v_{9}$ & $v_{10}$ & $v_{11}$ & $v_{12}$ & $v_{13}$ \\
\hline$x$ & 4.0 & 0.0 & 0.0 & 4.0 & 0.0 & 0.0 & -5.0 & -3.0 & -3.0 & -5.0 & 1.0 & -1.0 & -3.0 \\
$y$ & 1.0 & 1.0 & 1.0 & 1.0 & -11.0 & -11.0 & -5.0 & -11.0 & -11.0 & -5.0 & -4.0 & -10.0 & -4.0 \\
$z$ & 7.0 & 7.0 & 15.0 & 15.0 & 15.0 & 7.0 & 7.0 & 7.0 & 15.0 & 15.0 & 1.0 & 1.0 & 1.0 \\
\hline
\end{tabular}

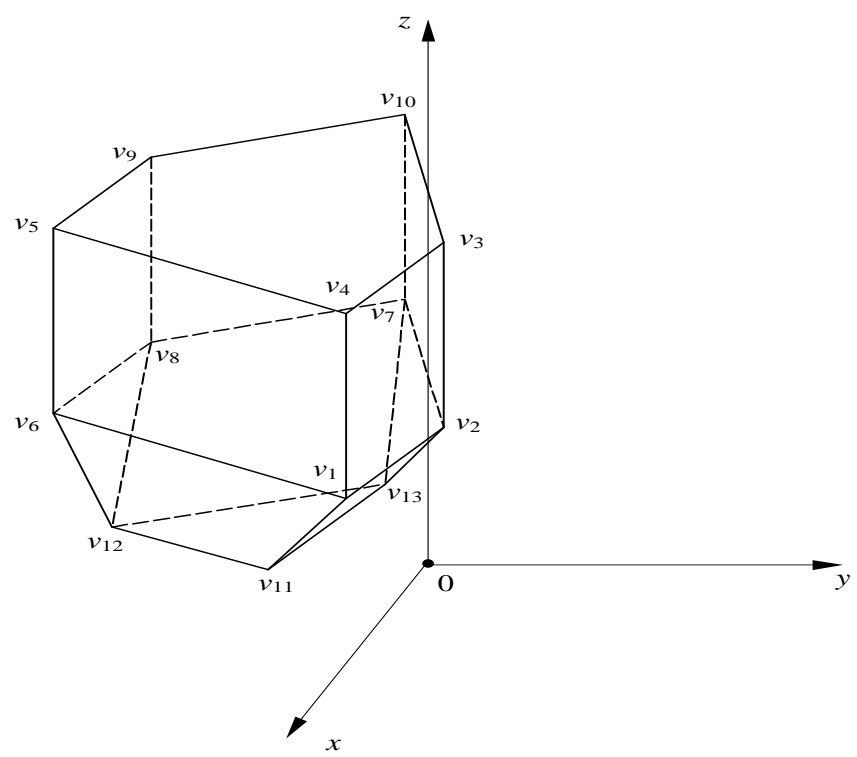

Fig. 5. $\Phi$-function of two polytopes

The following linear functions result for $\gamma_{12}: f_{1}(u)=y-1, f_{2}(u)=$ $3 x-y-11, \quad f_{3}(u)=-3 x-y-20, \quad f_{4}(u)=z-15, \quad f_{5}(u)=-z+1$, $f_{6}(u)=-6 y-z-59, f_{7}(u)=-36 x+30 y-7 z+19, f_{8}(u)=9 x-3 y-2 z-19$, $f_{9}(u)=6 y-5 z+39, \quad f_{10}(u)=-6 x+5 y-5, f_{11}(u)=-y-11, f_{12}(u)=$ $-18 x-6 y-7 z-71$.

Hence, $\Phi_{12}\left(u_{1}, u_{2}\right)=\max \left\{f_{1}\left(u_{2}-u_{1}\right), f_{2}\left(u_{2}-u_{1}\right), \ldots, f_{12}\left(u_{2}-u_{1}\right)\right\}$ is a $\Phi$-function for the two polytopes. Note that it is not normalized, even if the functions $f_{i}$ have a normalized coefficient vector. 
6. Algorithm for constructing $\gamma_{12}$. In this section we present an algorithmic description of the above computation of the surface $\gamma_{12}$ of two convex polytopes which are given in facet representation.

Let $h_{i}^{k}\left\langle v_{i 1}^{k}, v_{i 2}^{k}, \ldots, v_{i m_{i}}^{k}\right\rangle, i=1, \ldots, N_{k}$, denote the facets of $T_{k}, k=1,2$, and let $\Omega$ denote the set of vertices of $\gamma_{12} ; \varepsilon$ denotes a given tolerance.

\section{Algorithm}

$\Omega=\emptyset$.

1. Considering the facets of $T_{1}$ and a vertex, edge or facet of $T_{2}$

For $i:=1$ to $N_{1}$ do:

- Calculate the coefficients of $f_{i}^{1}: n_{i}^{1}=\left(A_{i}^{1}, B_{i}^{1}, C_{i}^{1}\right)^{T}=\left(v_{i 2}^{1}-v_{i 1}^{1}\right) \times$ $\left(v_{i 3}^{1}-v_{i 1}^{1}\right), D_{i}^{1}=-\left(n_{i}^{1}\right)^{T} v_{i 1}^{1}$ where "×" denotes vector product.

- Compute $J_{i}^{2}=\left\{j^{\prime} \in\left\{1, \ldots, M_{2}\right\}: f_{i}^{1}\left(v_{j^{\prime}}^{2}\right)=\min \left\{f_{i}^{1}\left(v_{j}^{2}\right): j=\right.\right.$ $\left.\left.1, \ldots, M_{2}\right\}\right\}$ and let $J_{i}^{2}=\left\{j_{1}, \ldots, j_{\chi}\right\}$.

- If $\chi=1$ then compute the facet $q=\left\langle v_{i 1}^{1}-v_{j_{1}}^{2}, \ldots, v_{i m_{i}}^{1}-v_{j_{1}}^{2}\right\rangle$ of $\gamma_{12}$. Otherwise, select a projection coordinate plane as follows:

— the $x y$-plane if $\left|C_{i}^{1}\right|=\max \left\{\left|A_{i}^{1}\right|,\left|B_{i}^{1}\right|,\left|C_{i}^{1}\right|\right\}$,

- the $z x$-plane if $\left|B_{i}^{1}\right|=\max \left\{\left|A_{i}^{1}\right|,\left|B_{i}^{1}\right|,\left|C_{i}^{1}\right|\right\}$,

- the $y z$-plane if $\left|A_{i}^{1}\right|=\max \left\{\left|A_{i}^{1}\right|,\left|B_{i}^{1}\right|,\left|C_{i}^{1}\right|\right\}$.

- If $\chi=2$ then project facet $h_{i}^{1}$ of $T_{1}$ and edge $e^{2}$ of $T_{2}$ with endpoints $v_{j_{1}}^{2}$ and $v_{j_{2}}^{2}$ onto the selected plane and construct the sequence of vertices of $q$ in accordance with item 2 of Section 4.

- If $\chi>2$ then project facet $h_{i}^{1}$ of $T_{1}$ and facet $h_{j}^{2}$ of $T_{2}$ (determined by $J_{i}^{2}$ ) onto the selected coordinate plane. Construct facet $q$ of $\gamma_{12}$ in accordance with item 3 of Section 4 .

- Update the vertex set $\Omega$ of $\gamma_{12}$ and save the representation of $q$ with respect to $\Omega$.

2. Considering the facets $h_{j}^{2}$ of $T_{2}$ and a vertex or edge of $T_{1}$

For $j=1$ to $N_{2}$ do:

- Calculate the coefficients of $f_{j}^{2}$ in analogy to step 1.

- Compute $J_{j}^{1}=\left\{i^{\prime} \in\left\{1, \ldots, M_{1}\right\}: f_{j}^{2}\left(v_{i^{\prime}}^{1}\right)=\min \left\{f_{j}^{2}\left(v_{i}^{1}\right): i=\right.\right.$ $\left.\left.1, \ldots, M_{1}\right\}\right\}$ and let $J_{j}^{1}=\left\{i_{1}, \ldots, i_{\chi}\right\}$.

- If $\chi=1$ then compute the facet $q=\left\langle v_{j m_{j}}^{2}-v_{i_{1}}^{1}, \ldots, v_{j 1}^{2}-v_{i_{1}}^{1}\right\rangle$.

Note that the circuit direction of the vertices in $q$ has to be opposite to that in $h_{j}^{2}$.

- If $\chi=2$ then project facet $h_{j}^{2}$ of $T_{2}$ and edge $e^{1}$ of $T_{1}$ with endpoints $v_{i_{1}}^{1}$ and $v_{i_{2}}^{1}$ onto a coordinate plane selected in analogy to step 1 . Construct the facet $q=\left\langle p_{1}, \ldots, p_{r}\right\rangle$ of $\gamma_{12}$ in accordance with item 5 
of Section 4. If the direction of the normal $n=\left(p_{2}-p_{1}\right) \times\left(p_{3}-p_{1}\right)$ is not opposite to that of $n_{j}^{2}$, then change the circuit orientation of $q$.

- If $\chi \leq 2$ then update the vertex set $\Omega$ of $\gamma_{12}$ and save the representation of $q$ with respect to $\Omega$.

3. Considering the edges $e_{i}^{1}$ of $T_{1}$ and the edges $e_{j}^{2}$ of $T_{2}$

Form a list of edges $e_{i}^{1}$ of $T_{1}$ specified by their end-vertices $v_{i_{1}}^{1}$ and $v_{i_{2}}^{1}$, $i=1, \ldots, K_{1}$, and a list of edges $e_{j}^{2}$ of $T_{2}$ with end-vertices $v_{j_{1}}^{2}$ and $v_{j_{2}}^{2}$, $j=1, \ldots, K_{2}$.

For $i=1$ to $K_{1}$ do:

For $j=1$ to $K_{2}$ do:

- If $\left\|e_{i}^{1}\right\|^{2} \cdot\left\|e_{j}^{2}\right\|^{2}-\left(\left(e_{i}^{1}\right)^{T} e_{j}^{2}\right)^{2}<\varepsilon$ then the two edges are parallel, no facet can be obtained. Otherwise calculate the normal vector $n=e_{i}^{1} \times e_{j}^{2}$ of the plane $H$ which is determined by $e_{i}^{1}$ and $e_{j}^{2}$.

- Verification whether $H$ is a separating plane

Set sep = true. Compute $J_{1}=\left\{r \in\left\{1, \ldots, M_{1}\right\}: n^{T} v_{r}^{1}=n^{T} v_{i_{1}}^{1}\right\}$, $\mu_{1}=\max \left\{n^{T} v_{r}^{1}: r=1, \ldots, M_{1}\right\}, \nu_{1}=\min \left\{n^{T} v_{r}^{1}: r=1, \ldots, M_{1}\right\}$. If $\nu_{1}<n^{T} v_{i_{1}}^{1}<\mu_{1}$ or $\operatorname{card}\left(J_{1}\right)>2$ then sep $=$ false.

If sep $=$ true then compute $J_{2}=\left\{s \in\left\{1, \ldots, M_{2}\right\}: n^{T} v_{s}^{2}=n^{T} v_{j_{1}}^{2}\right\}$, $\mu_{2}=\max \left\{n^{T} v_{s}^{2}: s=1, \ldots, M_{2}\right\}, \nu_{2}=\min \left\{n^{T} v_{s}^{2}: s=1, \ldots, M_{2}\right\}$. If $\nu_{2}<n^{T} v_{j_{1}}^{2}<\mu_{2}$ or card $\left.J_{2}\right)>2$ then sep $=$ false.

If $\nu_{1}=n^{T} v_{i_{1}}^{1}$ and $\nu_{2}=n^{T} v_{j_{1}}^{2}$ or if $\mu_{1}=n^{T} v_{i_{1}}^{1}$ and $\mu_{2}=n^{T} v_{j_{1}}^{2}$ then sep $=$ false.

- If sep $=$ true then calculate the facet $q$ of $\gamma_{12}$ in accordance with item 6 of Section 4, i.e. $q$ has the vertices $p_{1}=v_{i_{1}}^{1}-v_{j_{1}}^{2}, p_{2}=v_{i 2}^{1}-v_{j_{1}}^{2}$, $p_{3}=v_{i 2}^{1}-v_{j 2}^{2}$, and $p_{4}=v_{i_{1}}^{1}-v_{j 2}^{2}$. Let $v_{t}^{1}$ be any vertex of $T_{1}$ not belonging to $e_{i}^{1}$, and let $n^{\prime}=\left(p_{2}-p_{1}\right) \times\left(p_{3}-p_{1}\right)$ be a normal vector of $q$. If $\left(n^{\prime}\right)^{T} v_{t}^{1}>\left(n^{\prime}\right)^{T} v_{i_{1}}^{1}$ then change the circuit orientation of $q$. Update the vertex set $\Omega$ of $\gamma_{12}$ and save the representation of $q$ with respect to $\Omega$.

7. Considering two parallel facets. For completeness, in this section we describe in detail one possibility to compute a facet $q=q_{p t}$ of $\gamma_{12}$ which is determined by two parallel facets $h_{p}^{1} \subset T_{1}$ and $h_{t}^{2} \subset T_{2}$. Here the projection onto the $x y$-plane is considered; the other two cases are similar.

Let $h_{p}^{1}\left\langle v_{p 1}^{1}, v_{p 2}^{1}, \ldots, v_{p n}^{1}\right\rangle$ and $h_{t}^{2}\left\langle v_{t 1}^{2}, v_{t 2}^{2}, \ldots, v_{t m}^{2}\right\rangle$ be facets of $T_{1}$ (considered to be fixed) and $T_{2}$ (which is movable), respectively. The facets are assumed to be parallel, and the vertices $v_{p i}^{1}=\left(x_{p i}^{1}, y_{p i}^{1}, z_{p i}^{1}\right), i=1, \ldots, n$, and $v_{t j}^{2}=\left(x_{t j}^{2}, y_{t j}^{2}, z_{t j}^{2}\right), j=1, \ldots, m$, are given in the eigen coordinate systems. Projection of the facets onto the $x y$-plane yields polygons $\operatorname{pr}_{x y} h_{p}^{1}=$ 
$\left\langle u_{p 1}^{1}, u_{p 2}^{1}, \ldots, u_{p n}^{1}\right\rangle$ and $\operatorname{pr}_{x y} h_{t}^{2}=\left\langle u_{t 1}^{2}, u_{t 2}^{2}, \ldots, u_{t m}^{2}\right\rangle$, where $u_{p i}^{1}=\operatorname{pr}_{x y} v_{p i}^{1}=$ $\left(x_{p i}^{1}, y_{p i}^{1}\right), i=1, \ldots, n$, and $u_{t j}^{2}=\operatorname{pr}_{x y} v_{t j}^{2}=\left(x_{t j}^{2}, y_{t j}^{2}\right), j=1, \ldots, m$. It is assumed that the eigen coordinate systems coincide and that the circuit directions of the two polygons are counterclockwise. Then the construction of the facet $q$ which is formed by the parallel facets $h_{p}^{1}$ and $h_{t}^{2}$ can be realized as follows:

1. In order to obtain a first vertex of $q$, an initial pair of vertices $u_{p k}^{1}$ and $u_{t r}^{2}$ is chosen such that $x_{p k}^{1}=\min \left\{x_{p 1}^{1}, x_{p 2}^{1}, \ldots, x_{p n}^{1}\right\}, y_{p k}^{1}=\max _{i}\left\{y_{p i}^{1}\right.$ : $\left.x_{p i}^{1}=x_{p k}^{1}\right\}$ and $x_{t r}^{2}=\max \left\{x_{t 1}^{2}, x_{t 2}^{2}, \ldots, x_{t m}^{2}\right\}, y_{t r}^{2}=\min _{j}\left\{x_{t j}^{2}: x_{t j}^{2}=x_{t r}^{2}\right\}$.

This choice guarantees nonoverlapping of the polygons $\operatorname{pr}_{x y} h_{p}^{1}$ and $\operatorname{pr}_{x y} h_{t}^{2}$ when $\mathrm{pr}_{x y} h_{t}^{2}$ is translated by the vector $u_{p k}^{1}-u_{t r}^{2}$. As a result, the translated vertex $u_{t r}^{2}$ coincides with vertex $u_{p k}^{1}$ and the translated origin of the eigen coordinate system of the polygon $\mathrm{pr}_{x y} h_{t}^{2}$ is the projection of the first vertex of facet $q$ on the $x y$-plane.

As a consequence, the first vertex $v_{1}$ of $q$, determined by $v_{p k}^{1}$ and $v_{t r}^{2}$, is

$$
v_{1}=v_{p k}^{1}-v_{t r}^{2} \text {. }
$$

2. All subsequent vertices $v_{l}, l=2, \ldots, L$ (with $L \leq m+n$ ), of facet $q$ can be computed step by step based on analysing the current geometrical situation. In detail, let the current constructed vertex $v_{l}$ of $q$ be formed by $u_{p i}^{1}$ and $u_{t j}^{2}$. Then the next vertex $v_{l+1}$ of $q$ is determined in analogy to the two-dimensional case by a pair of vertices: either by $u_{p, i+1}^{1}, u_{t j}^{2}\left(\operatorname{shifting} T_{2}\right.$ along an edge of $T_{1}$ ), or by $u_{p i}^{1}, u_{t, j+1}^{2}$ (shifting $T_{2}$ along an edge of $T_{2}$ ), or by $u_{p, i+1}^{1}, u_{t, j+1}^{2}$ (shifting $T_{2}$ along parallel edges).

More formally, let

$$
\vartheta_{j}\left(x_{p i}^{1}, y_{p i}^{1}\right)=\left|\begin{array}{cc}
x_{p i}^{1}-x_{t j}^{2} & y_{p i}^{1}-y_{t j}^{2} \\
x_{t, j+1}^{2}-x_{t j}^{2} & y_{t, j+1}^{2}-y_{t j}^{2}
\end{array}\right| .
$$

If $\vartheta_{j}\left(x_{p i}^{1}, y_{p i}^{1}\right)=\vartheta_{j}\left(x_{p, i+1}^{1}, y_{p, i+1}^{1}\right)$ then $v_{l+1}=v_{p, i+1}^{1}-v_{t, j+1}^{2}$.

If $\vartheta_{j}\left(x_{p i}^{1}, y_{p i}^{1}\right)<\vartheta_{j}\left(x_{p, i+1}^{1}, y_{p, i+1}^{1}\right)$ then $v_{l+1}=v_{p i}^{1}-v_{t, j+1}^{2}$.

If $\vartheta_{j}\left(x_{p i}^{1}, y_{p i}^{1}\right)>\vartheta_{j}\left(x_{p, i+1}^{1}, y_{p, i+1}^{1}\right)$ then $v_{l+1}=v_{p, i+1}^{1}-v_{t j}^{2}$.

Finally, when vertex $v_{l+1}$ coincides with vertex $v_{1}$ then the construction of facet $q$ for $\gamma_{12}$ is terminated.

It is to be noted that in the case where an edge of $T_{2}$ is parallel to a facet of $T_{1}$, the algorithm is also applicable, now with $m=2$. The algorithm does not change significantly when facets $h_{p}^{1}$ and $h_{t}^{2}$ are projected onto the $y z$-plane or $z x$-plane. However the definition of $u_{p i}^{1}$ and $u_{t j}^{2}$ changes.

8. Concluding remarks. When dealing with $3 \mathrm{D}$ packing problems, tools like $\Phi$-functions are needed to describe or characterize the mutual position of two objects. The proposed method for computing a $\Phi$-function of 
two convex polytopes is based on a facet-oriented description of the polytopes. Generalizing it to the nonconvex case is still an open problem.

Acknowledgments. The authors are grateful to the anonymous referee for his/her helpful comments.

\section{References}

[1] H. Dyckhoff and U. Finke, Cutting and Packing in Production and Distribution, Physica-Verlag, Heidelberg, 1992.

[2] H. Dyckhoff, G. Scheithauer, and J. Terno, Cutting and packing, in: M. Dell'Amico, et al. (eds), Annotated Bibliographies in Combinatorial Optimization, Chap. 22, Wiley, Chichester, 1997, 393-412.

[3] J. Edmonds, A combinatorial representation for polyhedral surfaces, Amer. Math. Soc. Notices 7 (1960).

[4] L. Fejes Tóth, Lagerungen in der Ebene, auf der Kugel und im Raum, Grundlehren Math. Wiss. 65, Springer, Berlin, 1953.

[5] B. Grünbaum, Convex Polytopes, Interscience, London, 1967.

[6] A. Hartwig, Algebraic 3-D Modelling, A. K. Peters, Wellesley, MA, 1996.

[7] L. S. Health, P. K. Paripati and J. W. Roach, Representing polyhedra: Faces are better than vertices, Comput. Geom. 3 (1993), 327-351.

[8] G. Scheithauer and J. Terno, Modelling of packing problems, Optimization 28 (1993), 63-84.

[9] Yu. G. Stoyan, On one generalization of the dense allocation function, Dokl. Akad. Nauk Ukrain. SSR Ser. A 1980, no. 8, 71-74.

[10] -, Mathematical methods for geometric design, in: T. M. R. Ellis and O. J. Semenkoc (eds.), Advances in CAD/CAM (Leningrad, 1982), Amsterdam, 1983, 6786.

[11] Y. G. Stoyan and A. F. Loyko, Automation of arrangement design of engineering system of block design. I. Mathematical model, Engineering Simulation 15 (1998), $815-825$.

[12] -, 一, Automation of arrangement design of engineering system of block design. II. Computer aided layout design, ibid. 16 (1998), 101-109.

[13] Y. G. Stoyan and L. D. Ponomarenko, Minkowski's sum and the hodograph of the dense allocation vector function, Dokl. Akad. Nauk Ukrain. SSR Ser. A 1977, no. 10, 887-890 (in Russian).

[14] J. Terno, R. Lindemann und G. Scheithauer, Zuschnittprobleme und ihre praktische Lösung, Verlag Harry Deutsch, Thun und Frankfurt/Main, 1987.

Institute for Problems in Machinery

National Ukrainian Academy of Sciences

2/10 Pozharsky St.

Kharkov, 61046, Ukraine

E-mail: stoyan@ipmach.kharkov.ua sherom@kharkov.ua
Institute for Numerical Mathematics

Dresden University of Technology 01062 Dresden, Germany

E-mail: scheit@math.tu-dresden.de 